
\title{
3 Research S Suare \\ Does Patella Position Influence Soft Tissue Balance and Clinical Outcome in Minimally Invasive Total Knee Arthroplasty?
}

\section{Kaiyuan Liu}

Shanghai Tenth People's Hospital

Dong Yang

Shanghai Tenth People's Hospital

Tianyang $\mathrm{Xu}$

Shanghai Tenth People's Hospital

Wenwei Jiang

Shanghai Tenth People's Hospital

\section{Lin Fan}

Shanghai Tenth People's Hospital

Guodong Li ( $\square$ litrue2004@163.com )

Shanghai Tenth People's Hospital Affiliated to Tongji University

\section{Research article}

Keywords: Minimally invasive total knee arthroplasty, patellar position, soft tissue balance, clinical effect

Posted Date: March 9th, 2020

DOl: https://doi.org/10.21203/rs.3.rs-16382/v1

License: (1) This work is licensed under a Creative Commons Attribution 4.0 International License.

Read Full License 


\section{Abstract}

Purpose: We hypothesized that patellar positioning during minimally invasive total knee arthroplasty (MIS-TKA) would affect soft tissue balance and postoperative outcome.

Methods: From December 2018 to February 2019, 55 patients receiving primary MIS-TKA were enrolled. The gap-balance technique was used, with patients randomly assigned to undergo osteotomy and balance of soft tissue with the patella reduced (group $A ; n=27$ ) or with the patella subluxated (group $B ; n$ $=28$ ). The soft tissue balance achieved with the patella reduced and subluxated were compared. Femoral prosthesis rotation, mechanical femoral axis-to-tibial axis angle, Knee Society Score (KSS), pain score, and range of flexion were compared between the groups. Follow-up was for 6 months.

Results: The flexion gap and the varus angle were significantly greater after patella reduction than before reduction, but the extension joint gap and varus angle were comparable before and after patella reduction. The femoral prosthesis tended to be internally rotated in group $B$. Range of flexion was better in the group A than in group B at 1 month after surgery, but the differences were not significant at 3 and 6 months. KSS and pain score were comparable between the groups after surgery.

Conclusion: During MIS-TKA, as far as possible, soft tissue balance should be achieved with the patella reduced; otherwise, the femoral prosthesis may be installed more internally and, after patella reduction, the flexion gap and varus would increase.

\section{Introduction}

Total knee arthroplasty (TKA) is widely used to treat end-stage knee arthritis $[6,8]$. Success of surgery depends on accurate osteotomy, accurate reconstruction of lower limb alignment, and achievement of the correct soft tissue balance. While the accuracy of osteotomies and prostheses placement has improved vastly with the introduction of computer navigation and three-dimensional (3D) printed prostheses, achieving soft tissue balance still depends on the surgeon's subjective assessment [7, 13]; too much soft tissue laxity can lead to joint instability and possibly even revision surgery, whereas excessive tightness will interfere with postoperative functional training and lead to dysfunction and reduced life of the prosthesis $[17,21,22]$.

For optimum soft tissue balance, the surgeon must obtain the same extension and flexion gaps and the same medial and lateral gaps [25]. Usually, these balances are obtained without reduction of the patella. However, more and more authors are stressing the need to assess soft tissue balance under physiological conditions, that is, with the patella reduced. In conventional TKA, the patella is everted during the operation; this eversion affects soft tissue balance, but exactly how much impact it has is still debated [16]. The effect of patellar eversion on the gap may vary with the type of prosthesis being used-i.e., posterior-cruciate retaining (CR) or posterior-cruciate substituting (PS). The effect on postoperative clinical function is also not yet clear. 
Minimally invasive total knee arthroplasty (MIS-TKA) has provided good clinical results [15, 23]. During MIS-TKA, the patella is not everted. However, the preservation of the extensor mechanism and the limited exposure actually leads to greater tension on the soft tissues than patellar eversion [19]. The lateral dislocation of the patella itself also has major impact on the soft tissue balance.

The purpose of this study was 1) to determine the effect of different patella positions on soft tissue balance during MIS-TKA and 2) to evaluate the difference in clinical effectiveness of TKA between patients achieving soft tissue balance with the patella in different positions.

\section{Materials And Methods}

\section{Patients}

From among the 81 patients who underwent MIS-TKA at our center between December 2018 and February 2019, we recruited 55 patients for this randomized clinical trial. Patients were eligible for inclusion if they 1 ) had osteoarthritis, with Kellgren-Lawrence (K-L) grade 3-4; 2) were undergoing primary TKA; and 3) had varus or neutral knee. Patients were excluded if they had 1) rheumatoid arthritis, 2) history of surgery or infection of the knee, 3) ligament injury of the knee, 4) valgus deformity, or 5) severe bone defects that might require insertion of pads. A total of 26/81 patients were excluded: 16 patients with K-L grade 2 severity, 3 with rheumatoid arthritis, 2 with severe varus knees and obvious bone defect, and 5 with valgus deformity. The remaining 55 patients were randomly separated into two groups: one group was assigned to receive patellar reduction (group $A ; n=27$ ) and the other to receive patellar subluxation (group $B ; \mathrm{n}=28$ ) during surgery.

This study was approved by the hospital ethics committee, and written informed consent was obtained from each patient. Operations were performed by the same senior surgeon. The patients and the staff who assessed postoperative efficacy were blinded to the intervention.

\section{General preoperative and postoperative measures}

Anteroposterior long-leg standing radiograph was taken to guide the selection of the valgus angle of the femoral component. Computed tomography (CT) scan of the knee was also acquired preoperatively. An antibiotic (second-generation cephalosporin) was routinely administered intravenously 20 minutes before surgery. The postoperative protocol for pain relief was the same for all patients. There was no drainage after surgery. The long-leg standing radiograph and CT of the operated knee were repeated at 5-7 days after operation. All patients received the same postoperative rehabilitation.

\section{Surgical technique}

A gap-balance technique was used in the operation [4]. All patients received a PS prosthesis (GENESIS II; Smith \& Nephew, USA). The patient was placed in the supine position, and an air tourniquet applied at $250 \mathrm{~mm} \mathrm{Hg}$; the tourniquet was loosened immediately after prosthesis implantation. Midline skin incision and the midvastus approach were used to expose the knee without everting the patella. The anterior 
cruciate ligament and meniscus were excised, and the distal femur was exposed. According to the preoperative long-leg standing radiograph, the valgus angle of the femoral component $\left(5^{\circ}-7^{\circ}\right)$ was selected. An intramedullary guide was used to perform distal femoral osteotomy. After adequate exposure, all osteophytes and menisci were removed. Then, the tibia was subluxated anteriorly. Osteotomy was performed using an extramedullary guide to ensure that each cut was perpendicular to the mechanical axis of the tibia in the coronal plane. The posterior inclination along the sagittal plane and the rotational alignment were checked. After the osteotomy, the gap balance was assessed with a conventional spacer block. The assessment was performed after reduction of the patella in group $A$ patients, and with the patella in the subluxated state in group B patients. If there was asymmetry, appropriate release of soft tissues was performed until a balanced extension gap was obtained in the coronal plane.

The femoral sizing guide was closely applied to the osteotomy surface of the distal femur, and the size of the osteotomy module was determined according to the anteroposterior diameter of the femur. A 3.2-mm drill bit was used to drill through the nail hole on the femoral sizing guide, which was then removed. Next, the external osteotomy angle of the femoral posterior condyle was redetermined by the gravity method, with an assistant lifting the patient's thigh to make the tibia sag naturally. In group A, the patella was maintained in the reduced position while the knee was flexed to $90^{\circ}$. In group B, the patella was first subluxated and then the knee was flexed to $90^{\circ}$. Then the matching rectangle was used to record the line (Figure 1). The positioning hole of the osteotomy module was adjusted according to the scribe line. Then, the anteroposterior cutting block was placed on the osteotomy surface of the distal femur, fixed in place with three nails. A complete femoral osteotomy was performed after confirming that the flexion gap was satisfactory. A femoral trial prosthesis was installed. The matching (left or right) offset-type tensor was selected and fixed to the proximal tibia, and then fitted to the femoral trial prosthesis. The joint distraction force was set at $40 \mathrm{lb}$ in each patient. Gap length and varus ligament imbalance were measured at $0^{\circ}$ and $90^{\circ}$ of knee flexion. In group A patients the measurements were only made with the patella reduced, whereas in group B patients the measurements were made with the patella laterally shifted and then, again, with the patella reduced.

\section{Observation indices}

The following were recorded:

1. Basic information (sex, age, height, weight, left or right knee)

2. Femoral rotational alignment and femoral prothesis rotation angle. On the CT scan of the knee, the angle between the surgical transepicondylar axis and the posterior condyle line of the femur was the femoral rotational alignment, and the angle between the surgical transepicondylar axis and posterior condyle line of the prosthesis was femoral prosthesis rotation angle (Figure 2). External rotation was indicated with the "plus" sign, and internal rotation with the "minus" sign. The surgical transepicondylar axis represents the axis of rotation of the original femur of the knee joint. The posterior condyle line of the prosthesis can be regarded as the axis of rotation of the prosthesis. The 
femoral prothesis rotation angle reflects the coincidence of the axis of rotation of the prosthesis with the axis of rotation of the original femur.

3. Mechanical femoral axis-to-tibial axis angle (mFTA). The angle between the mechanical axis of the tibia and the mechanical axis of the femur before and after surgery were measured on the long-leg standing radiographs; the target angle after surgery was $0^{\circ} \pm 3^{\circ}$.

4. Offset-Repo-Tensor measurement of joint gap and varus angle (Figure 3). As recommended in previous studies [18], the joint gap length and varus angle were measured with the knee in full extension and $90^{\circ}$ of flexion at. A torque wrench was used to ensure that the joint distraction force was set at $40 \mathrm{lb}$ (Figure 3)

5. Knee Society Score. KSS score was assessed before surgery and at 1 month, 3 months, and 6 months after surgery. The KSS (maximum possible score 100) takes into consideration pain, activity, and stability and reflects knee function.

6. Pain assessment. A visual analog scale (VAS; ranging from 0 to 10) was used to evaluate knee pain before surgery and at and 1 month, 3 months, and 6 months after surgery.

7. Range of flexion. Maximum flexion achievable in the knee was recorded before surgery and at 1 month, 3 months, and 6 months after surgery.

\section{Statistical analysis}

SPSS 25.0 (IBM Corp., Armonk, NY, USA) was used for statistical analysis. Differences between groups in age, body mass index (BMI), femoral rotational alignment, femoral prosthesis rotational alignment, joint gap, varus angle, KSS, and VAS were compared using the independent-samples $t$ test. The joint gap and varus angle in group $B$ before and after reduction were compared using the paired sample $t$ test. Pearson chi-square test was used to compare categorical variables (sex distribution and side affected). Statistical significance was at $P \leq 0.05$.

\section{Result}

All 55 patients completed 6 months of follow-up. The two groups were comparable with regard to age, sex distribution, BMI, side affected (left or right), preoperative KSS, preoperative range of flexion, preoperative VAS, femoral rotational alignment, and preoperative mFTA $(P>0.05$; Table 1$)$. The operation was uneventful in all patients. Postoperatively, one patient in group $A$ developed deep venous thrombosis (revealed on B-ultrasonography) but improved after anticoagulation; one patient in group B had necrosis of the surgical wound but recovered after 5 weeks of dressing changes.

\section{Intraoperative soft tissue balance}

\section{Comparison between group $A$ and group $B$}

The soft tissue balance was within a satisfactory range for all patients. There were no significant differences between the groups in joint gap and varus angle in extension or in flexion ( $P>0.05$; Table 2$)$. 
In $90^{\circ}$ of flexion, the joint gap was $11.0 \pm 0.3 \mathrm{~mm}$ after patella reduction vs. $10.5 \pm 0.3 \mathrm{~mm}(t=4.18, \mathrm{P}<$ 0.01 ) before patella reduction, and the gap varus angle was $2.3^{\circ} \pm 0.4^{\circ}$ after reduction vs. $1.5^{\circ} \pm 0.3^{\circ}$ before reduction $(t=7.64, \mathrm{P}<0.01)$. With the knee extended, the differences in joint gap and varus angle before and after patella reduction were not statistically significant $(P>0.05$; Table 3$)$.

\section{Postoperative evaluation}

Imaging evaluation

The rotational angle of the femoral component was $0.24^{\circ} \pm 1.3^{\circ}$ in group $A$ and more than $-0.49^{\circ} \pm 1.2^{\circ}$ in group $\mathrm{B}$; the difference was statistically significant $(t=2.12, \mathrm{P}=0.039)$, which is more obvious in the scatter plot of femoral prothesis rotation angle (Figure 4$)$. The angle of the lower limb mechanical axis was corrected to within $0^{\circ} \pm 3^{\circ}$ in both groups $(P>0.05$; Table 4$)$.

KSS and VAS

The KSS and VAS scores were significantly improved after surgery in both groups. The mean KSS scores at 1 month, 3 months, and 6 months after surgery were $73.3 \pm 8.8,80.4 \pm 10.0$, and $84.9 \pm 6.2$, respectively, in group A vs. $72.4 \pm 8.0,80.4 \pm 11.8$, and $85.1 \pm 8.0$, respectively, in group B; the differences between the two groups were not statistically significant $(P>0.05$; Figure 5$)$. The VAS scores at 1 month, 3 months, and 6 months after surgery were $4.0 \pm 1.8,1.6 \pm 0.9$, and $1.2 \pm 0.8$, respectively, in group A vs. $4.0 \pm 1.5,1.7 \pm 1.2$, and $1.2 \pm 0.9$, respectively, in group $B$; the differences between the two groups were not statistically significant $(P>0.05$; Figure 6$)$.

\section{Range of flexion}

Mean range of flexion at 1 month after surgery was significantly better in group A than in group B: $109.6^{\circ}$ $\pm 8.5^{\circ}$ vs. $104.9^{\circ} \pm 8.6^{\circ}(t=2.05, \mathrm{P}=0.046$; Figure 7$)$. However, the range of flexion at 3 months and 6 months after surgery was comparable between the groups $(P>0.05$; Figure 7$)$.

\section{Discussion}

The surgical approach and the degree of exposure influences soft tissue tension and thus affects the surgeon's judgment of soft tissue balance [19]. This study showed that the joint gap at $90^{\circ}$ of flexion was significantly smaller with the patella shifted laterally than with the patella reduced; the reduction of the lateral compartment space was also more obvious. This proves that subluxation of the patella intraoperatively will lead to underestimation of the joint gap at flexion, and that the soft tissue balance is more reliably estimated after the patella is reduced. Yoon et al. compared the changes in the extension and flexion gaps with the patella everted [26], subluxated, and reduced in patients undergoing traditional TKA. They found that the flexion gap was significantly smaller, and the valgus greater, when the patella was everted than when it was subluxated or reduced. The joint gap and varus angle were comparable 
with patellar subluxated and with patella reduced. The extension gap was not significantly affected by patella position. A cadaveric experiment by Gejo also indicated that eversion of the patella during traditional TKA might lead to underestimation of the flexion gap [10]. It should be noted, of course, that the effect of patella position on soft tissue balance varies with the type of prosthesis used [2]. All of our patients received the PS prosthesis.

As mentioned earlier, soft tissue tension is relatively high during MIS-TKA; subluxation of the patella further increases the tension. In fact, the patella subluxation will result in greater soft tissue tension than is seen with patella eversion in conventional TKA. The choice of the surgical approach (i.e., quadricepssparing (QS), midvastus, subvastus, or limited parapatellar approaches) influences the soft tissue tension. Oka et al. [20] compared the QS approach with the limited parapatellar approach and found that subluxation of the patella results in underestimation of the flexion gap in both groups of patients, though the effect was significantly more in patients receiving the QS approach. The varus angle changed significantly only in the QS group, probably due to the greater soft tissue tension generated when the patella was subluxated in the QS approach. Our study, in which the midvastus approach was used for all patients, also showed reduction of joint gap and varus angle after patellar subluxation. And most previous study used the measured resection in operations while a modified gap balance technique was applied intraoperatively in ours. Keyser found that the effect of patellar subluxation on the flexion gap was mainly on the lateral gap in the MIS-TKA approach [5]; this is consistent with the increase of the lateral gap and the increase of the varus angle after patellar reduction seen in in our study. According to Gejo, the flexion gap is also affected by patellar ligament tension, which may vary between patients [9]. Therefore, to obtain accurate soft tissue balance, especially the gap balance in the flexion position, and to get closer to the preoperative soft tissue state, soft tissue balance must be judged with the patella reduced. According to Kamei, reduction in the bone mass effect of the distal femur after femoral osteotomy results in loosening of the patellar ligament was loosened and, consequently, to increase in the joint gap [14]. To counteract this effect, we chose to do the measurement after the femoral prothesis was installed.

Recovery of the correct coronal limb alignment and femoral prosthesis rotation alignment are essential for good postoperative function. Poor coronary limb alignment can lead to residual deformities and accelerated wear of the prosthesis, while poor rotational alignment of the femoral prosthesis can cause imbalance of the flexion gap and poor patellar tracking, which will eventually affect knee function $[1,12]$. In this study we found that although there was no significant difference in the MFTA, the femoral prothesis was more internally rotated in group $B$ patients. This was because when the patellar is subluxated at $90^{\circ}$ flexion the reduction in gap is greater laterally than medially, which results in a reduction of the varus angle. As the tibial plateau was taken as a reference to perform osteotomy of femoral posterior condylar with the gap-balance technique, the line parallel to tibial plateau was more internal, which eventually caused the internal installment of femoral prothesis (Figure 8). Therefore, we believe that in MIS-TKA, although patella position does not affect the reconstruction of the coronal limb alignment after surgery, the soft tissue balance achieved with the patella subluxated can lead to an increase in the internal rotation of the femoral prosthesis. 
In this study, KSS, VAS, and range of flexion showed significant improvement at 1 month after surgery. The improvement in the range of flexion was better in group $A$ at the first follow-up visit; this may be related to better patellar tracking and physiological balance of soft tissue in this group. No significant differences in KSS and VAS were observed between the two groups. Many factors other than the surgical technique affect patient satisfaction; these may include the patient's medical history, pain threshold, mental status, and expectations $[3,11,24]$. All of these also deserve the attention of the surgical team before and after surgery.

There are some limitations in this study. First, patients with valgus were excluded and most patients were women; these factors may have biased the results. Second, previous literature indicates that the application of a tourniquet may affect the extensor mechanism; this could theoretically affect the balance of the intraoperative gap, we could not avoid the use of tourniquets in this study. Finally, the sample size was small and the follow-up time was short. No conclusions can be drawn on prosthetic wear and survival rates.

\section{Conclusions}

The impact of the patellar position on the flexion gap should be considered when assessing soft tissue balance during MIS-TKA. As far as possible, soft tissue balance must be achieved with the patellar reduced; otherwise, the femoral prosthesis may be installed relatively internally and, after patella reduction, the flexion gap and the varus would increase.

\section{Abbreviations}

MIS-TKA囚minimally invasive total knee arthroplasty;

TKA囚Total knee arthroplasty; KSS₫Knee Society Score

CR囚posterior-cruciate retaining; PS: posterior-cruciate substituting

CT: Computed tomography; mFTA: Mechanical femoral axis-to-tibial axis angle

VAS: visual analog scale; BMI: body mass index

\section{Declarations}

\section{Availability of data and materials}

The datasets used and analyzed during the current study are available from the corresponding author on reasonable request.

\section{Authors' contributions}


KYL and GDL conceived and designed the study. DY and XTY collected and processed the data. KYL and DY wrote the paper. KYL, DY $\triangle T Y X, L F$ and WWJ reviewed and edited the manuscript. All authors read and approved the final manuscript.

\section{Ethics approval and consent to participate}

All procedures performed in studies involving human participants were in accordance with the ethical standards of the institutional and/or national research committee and with the 1964 Helsinki declaration and its later amendments or comparable ethical standards. This study was approved by the institutional review board of Shanghai Tenth People's Hospital.

\section{Consent for publication}

Not applicable

\section{Competing interests}

The authors declare that they have no competing interests.

\section{Funding}

This work was supported by the National Natural Science Foundation of China (NSFC), grant number (81572632).

\section{Acknowledgements:}

We really appreciate the colleagues from our department of psychiatry for helping to solve related issues.

\section{References}

1. Abdel MP, Ollivier M, Parratte S, Trousdale RT, Berry DJ, Pagnano MW (2018) Effect of Postoperative Mechanical Axis Alignment on Survival and Functional Outcomes of Modern Total Knee Arthroplasties with Cement: A Concise Follow-up at 20 Years. J Bone Joint Surg Am 100: 472-478

2. Aunan E, Kibsgard T, Rohrl SM (2017) Minimal effect of patella eversion on ligament balancing in cruciate-retaining total knee arthroplasty. Arch Orthop Trauma Surg 137: 387-392

3. Culliton SE, Bryant DM, Overend TJ, MacDonald SJ, Chesworth BM (2012) The relationship between expectations and satisfaction in patients undergoing primary total knee arthroplasty. J Arthroplasty 27: 490-492

4. Daines BK, Dennis DA (2014) Gap balancing vs. measured resection technique in total knee arthroplasty. Clin Orthop Surg 6: 1-8

5. De Keyser W, Beckers L (2010) Influence of patellar subluxation on ligament balancing in total knee arthroplasty through a subvastus approach. An in vivo study. Acta Orthop Belg 76: 799-805 
6. Dusad A, Pedro S, Mikuls TR, Hartman CW, Garvin KL, O'Dell JR, Michaud K (2015) Impact of Total Knee Arthroplasty as Assessed Using Patient-Reported Pain and Health-Related Quality of Life Indices: Rheumatoid Arthritis Versus Osteoarthritis. Arthritis Rheumatol 67: 2503-2511

7. Dutton AQ, Yeo SJ (2009) Computer-assisted minimally invasive total knee arthroplasty compared with standard total knee arthroplasty. Surgical technique. J Bone Joint Surg Am 91 Suppl 2 Pt 1: 116130

8. Evans JT, Whitehouse MR (2019) Partial versus total knee replacement for knee osteoarthritis. Lancet 394: 712-713

9. Gejo R, Morita Y, Matsushita I, Sugimori K, Kimura T (2008) Joint gap changes with patellar tendon strain and patellar position during TKA. Clin Orthop Relat Res 466: 946-951

10. Gejo R, McGarry MH, Jun BJ, Hofer JK, Kimura T, Lee TQ (2010) Biomechanical effects of patellar positioning on intraoperative knee joint gap measurement in total knee arthroplasty. Clin Biomech (Bristol, Avon) 25: 352-358

11. Greene KA, Harwin SF (2011) Maximizing patient satisfaction and functional results after total knee arthroplasty. J Knee Surg 24: 19-24

12. Hernandez-Vaquero D, Noriega-Fernandez A, Fernandez-Carreira JM, Fernandez-Simon JM, Llorens de los Rios J (2014) Computer-assisted surgery improves rotational positioning of the femoral component but not the tibial component in total knee arthroplasty. Knee Surg Sports Traumatol Arthrosc 22: 3127-3134

13. Hooper J, Schwarzkopf R, Fernandez E, Buckland A, Werner J, Einhorn T, Walker PS (2019) Feasibility of single-use 3D-printed instruments for total knee arthroplasty. Bone Joint J 101-B: 115-120

14. Kamei G, Murakami Y, Kazusa H, Hachisuka S, Inoue H, Nobutou H, Nishida K, Mochizuki Y, Ochi M (2011) Is patella eversion during total knee arthroplasty crucial for gap adjustment and soft-tissue balancing? Orthop Traumatol Surg Res 97: 287-291

15. Khanna A, Gougoulias N, Longo UG, Maffulli N (2009) Minimally invasive total knee arthroplasty: a systematic review. Orthop Clin North Am 40: 479-489, viii

16. Matsumoto T, Muratsu H, Tsumura N, Mizuno K, Kuroda R, Yoshiya S, Kurosaka M (2006) Joint gap kinematics in posterior-stabilized total knee arthroplasty measured by a new tensor with the navigation system. J Biomech Eng 128: 867-871

17. Matsumoto T, Kubo S, Muratsu H, Matsushita T, Ishida K, Kawakami Y, Oka S, Matsuzaki T, Kuroda Y, Nishida K, Akisue T, Kuroda R, Kurosaka M (2013) Different pattern in gap balancing between the cruciate-retaining and posterior-stabilized total knee arthroplasty. Knee Surg Sports Traumatol Arthrosc 21: 2338-2345

18. Nagai K, Muratsu H, Kanda Y, Tsubosaka M, Kamenaga T, Miya H, Matsushita T, Niikura T, Kuroda R, Matsumoto T (2018) Intraoperative soft tissue balance using novel medial preserving gap technique in posterior-stabilized total knee arthroplasty: comparison to measured resection technique. Knee Surg Sports Traumatol Arthrosc 26: 3474-3481 
19. Niki Y, Takeda Y, Kanagawa H, Iwamoto W, Matsumoto H, Enomoto H, Toyama Y, Suda Y (2012) Effects of four different surgical approaches on intra-operative joint gap in posterior-stabilized total knee arthroplasty. Knee Surg Sports Traumatol Arthrosc 20: 2026-2031

20. Oka S, Muratsu H, Matsumoto T, Kubo S, Maruo A, Miya H, Kuroda R, Kurosaka M (2012) The influence of patellar position on soft tissue balance in minimal incision total knee arthroplasty. Knee Surg Sports Traumatol Arthrosc 20: 1064-1068

21. Peters CL, Jimenez C, Erickson J, Anderson MB, Pelt CE (2013) Lessons learned from selective softtissue release for gap balancing in primary total knee arthroplasty: an analysis of 1216 consecutive total knee arthroplasties: AAOS exhibit selection. J Bone Joint Surg Am 95: e152

22. Sharkey PF, Hozack WJ, Rothman RH, Shastri S, Jacoby SM (2002) Insall Award paper. Why are total knee arthroplasties failing today? Clin Orthop Relat Res: 7-13

23. Shin YS, Kim HJ, Ko YR, Yoon JR (2016) Minimally invasive navigation-assisted versus conventional total knee arthroplasty: a meta-analysis. Knee Surg Sports Traumatol Arthrosc 24: 3425-3432

24. Von Keudell A, Sodha S, Collins J, Minas T, Fitz W, Gomoll AH (2014) Patient satisfaction after primary total and unicompartmental knee arthroplasty: an age-dependent analysis. Knee 21: 180-184

25. Yoon JR, Jeong HI, Oh KJ, Yang JH (2013) In vivo gap analysis in various knee flexion angles during navigation-assisted total knee arthroplasty. J Arthroplasty 28: 1796-1800

26. Yoon JR, Oh KJ, Wang JH, Yang JH (2015) Does patella position influence ligament balancing in total knee arthroplasty? Knee Surg Sports Traumatol Arthrosc 23: 2012-2018

\section{Tables}

Table 1 Comparison of baseline characteristics between the two groups

\begin{tabular}{|c|c|c|c|c|}
\hline & $\begin{array}{l}\text { Group A } \\
\mathrm{n}=27\end{array}$ & $\begin{array}{l}\text { Group B } \\
n=28\end{array}$ & $\mathrm{P}$ & $t / \chi^{2}$ \\
\hline tge, years, mean \pm SD (years) & $67.4 \pm 7.1$ & $68.4 \pm 8.6$ & 0.633 & \multirow{9}{*}{$\begin{array}{l}t=0.48 \\
\chi^{2}=0.34 \\
t=0.91 \\
\chi^{2}=0.16 \\
t=0.27 \\
t=0.68 \\
t=0.70 \\
t=10.1 \\
t=0.32\end{array}$} \\
\hline jex (female/male) & $22 / 5$ & $21 / 7$ & 0.561 & \\
\hline 3MI, mean \pm SD & $21.7 \pm 1.9$ & $22.1 \pm 1.6$ & 0.366 & \\
\hline _eft/right & $13 / 14$ & $14 / 14$ & 0.688 & \\
\hline ¿SS, mean \pm SD & $39.8 \pm 10.6$ & $40.5 \pm 8.0$ & 0.787 & \\
\hline Zange of flexion, mean $\pm \mathrm{SD}\left({ }^{\circ}\right)$ & $102.7^{\circ} \pm 9.3^{\circ}$ & $104.4^{\circ} \pm 9.4^{\circ}$ & 0.496 & \\
\hline JAS, mean $\pm \mathrm{SD}$ & $5.2 \pm 1.6$ & $5.2 \pm 1.5$ & 0.944 & \\
\hline${ }^{7}$ emoral rotational alignment, mean $\pm \mathrm{SD}\left({ }^{\circ}\right)$ & $3.6 \pm 1.1$ & $3.5 \pm 1.0$ & 0.795 & \\
\hline nFTA, mean $\pm \mathrm{SD}\left({ }^{\circ}\right)$ & $6.4 \pm 2.6$ & $6.6 \pm 2.7$ & 0.751 & \\
\hline
\end{tabular}

SD, standard deviation; BMI, body mass index; KSS, Knee Society Score; VAS, visual analog scale; mFTA, mechanical femoral axis-to-tibial axis angle

Table 2 Comparison of intraoperative balance between the two groups 


\begin{tabular}{lllll}
\hline Jroup & Group A & Group B & P & $t$ \\
& $\mathrm{n}=27$ & $\mathrm{n}=28$ & & \\
Jap at extension $(\mathrm{mm})$ & $10.4 \pm 0.3$ & $10.4 \pm 0.4$ & 0.838 & 0.21 \\
Jarus angle at extension $\left({ }^{\circ}\right)$ & $1.4 \pm 0.3$ & $1.4 \pm 0.3$ & 0.854 & 0.19 \\
Jap at flexion $\left(90^{\circ}\right)(\mathrm{mm})$ & $10.6 \pm 0.3$ & $10.5 \pm 0.3$ & 0.429 & 0.80 \\
Tarus angle at flexion $\left(90^{\circ}\right)\left(^{\circ}\right)$ & $1.6 \pm 0.4$ & $1.5 \pm 0.3$ & 0.654 & 0.45 \\
\hline
\end{tabular}

Values are the means \pm standard deviation

Table 3 Comparison of balance before and after reduction of patella in group B

\begin{tabular}{lllll}
\hline Position of patellar & Subluxation & Reduction & $\mathrm{P}$ & $t$ \\
\hline Gap at extension $(\mathrm{mm})$ & $10.4 \pm 0.4$ & $10.3 \pm 0.3$ & 0.179 & 1.38 \\
Varus angle at extension $\left(^{\circ}\right)$ & $1.4 \pm 0.3$ & $1.5 \pm 0.3$ & 0.130 & 1.56 \\
Gap at flexion $\left(90^{\circ}\right)(\mathrm{mm})$ & $10.5 \pm 0.3$ & $11.0 \pm 0.3$ & $<0.001 *$ & 4.18 \\
Varus angle at flexion $\left(90^{\circ}\right)\left(^{\circ}\right)$ & $1.5 \pm 0.3$ & $2.3 \pm 0.4$ & $<0.001^{*}$ & 7.64 \\
\hline
\end{tabular}

Table 4 Comparison of postoperative imaging parameters between the two groups

\begin{tabular}{llllc}
\hline Group & $\begin{array}{l}\text { Group A } \\
\mathrm{n}=27\end{array}$ & $\begin{array}{l}\text { Group B } \\
\mathrm{n}=28\end{array}$ & $\mathrm{P}$ & $t$ \\
\hline Femoral prosthesis rotational alignment $\left.^{\circ}\right)$ & $0.24 \pm 1.3$ & $-0.49 \pm 1.2$ & $0.039^{*}$ & 2.12 \\
mFTA $\left.^{\circ}\right)$ & $1.1 \pm 0.3$ & $1.0 \pm 0.2$ & 0.420 & 1.14 \\
\hline
\end{tabular}

Values are the means \pm standard deviation

mFTA, mechanical femoral axis-to-tibial axis angle

\section{Figures}

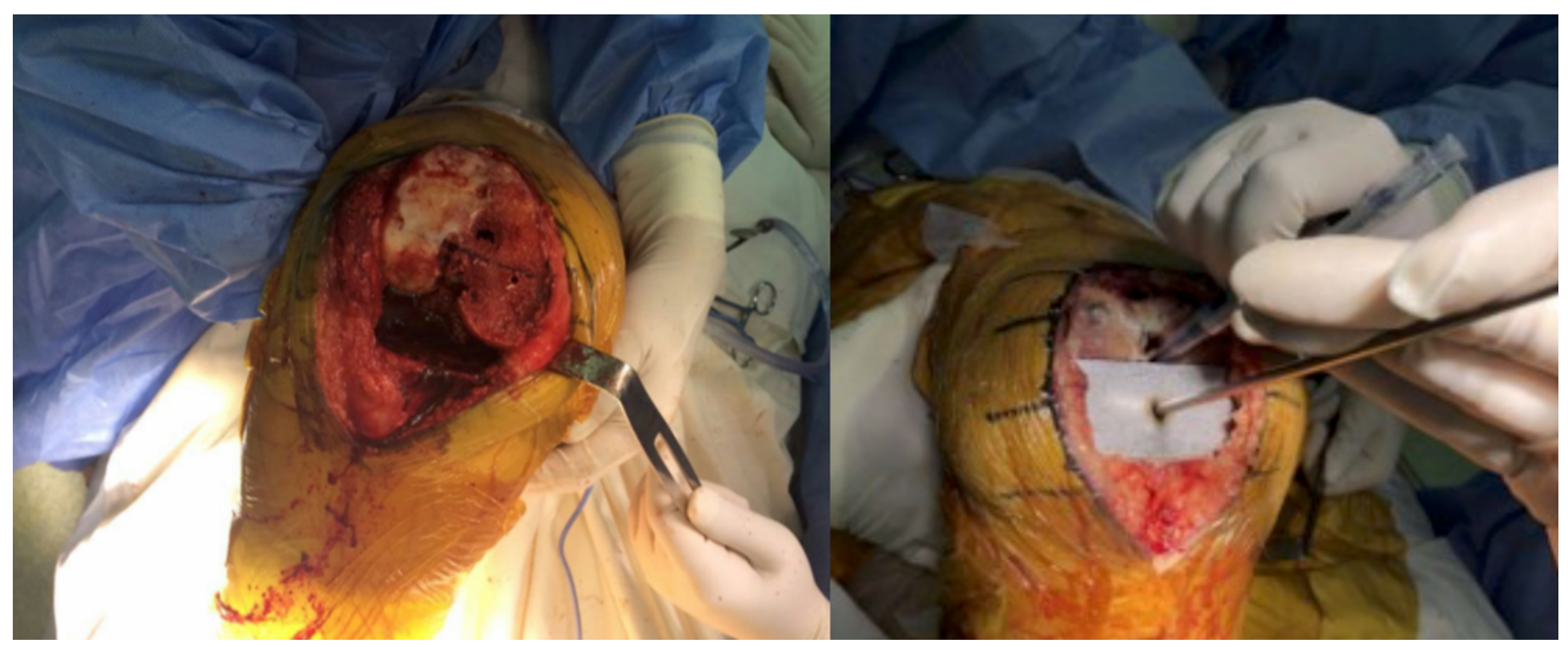

Figure 1 
The matching rectangle is used to record the line, and the external osteotomy angle of the femoral posterior condyle is adjusted according to the scribe line

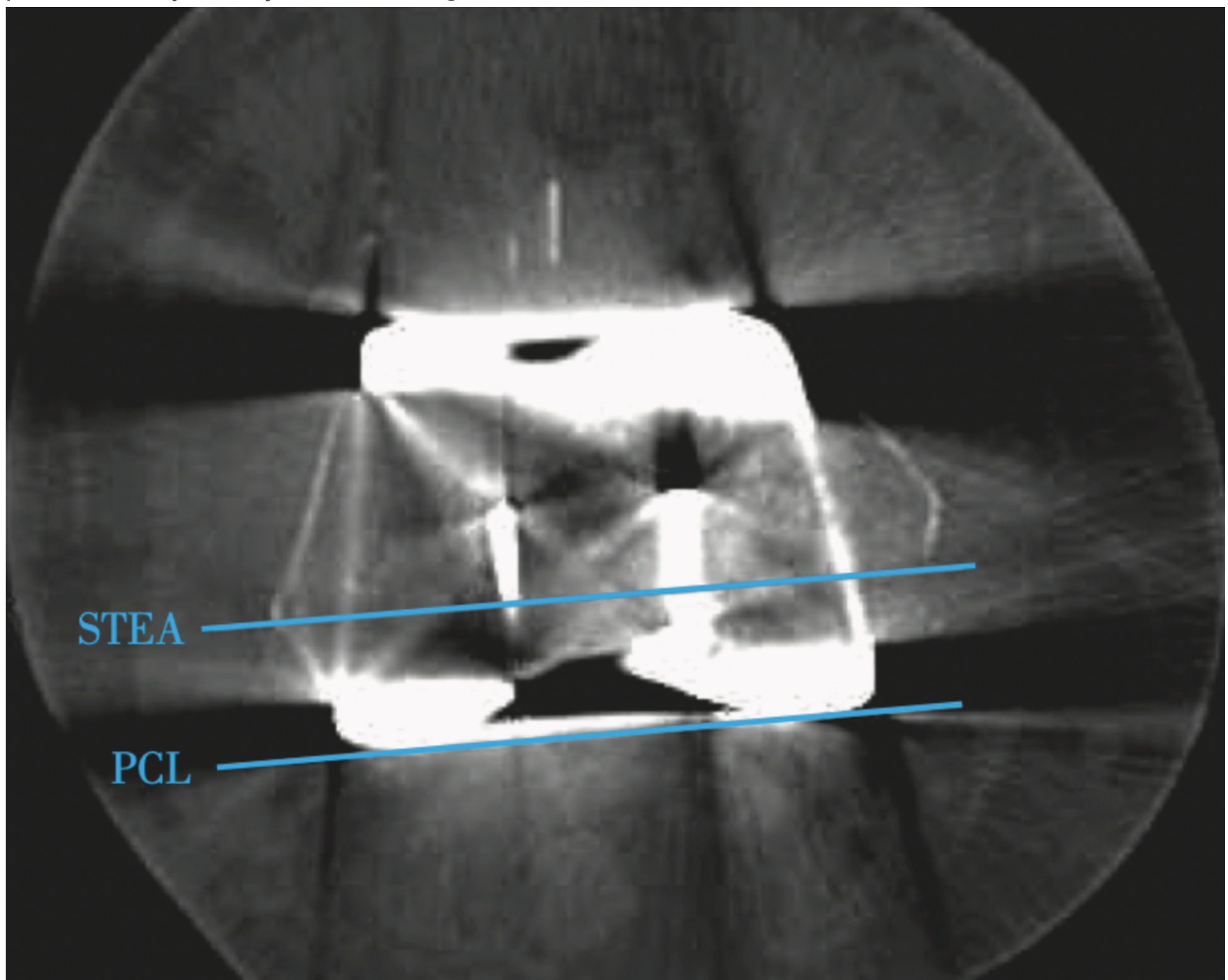

\section{Figure 2}

Postoperative CT of knee. The STEA (surgical transepicondylar axis) represents the axis of rotation of the original femur of the knee, while the PCL (posterior condyle line) of the prosthesis can be regarded as the axis of rotation of the prosthesis. The femoral prothesis rotation angle reflects the coincidence of the femoral prosthesis rotation axis with the surgical transepicondylar axis. 


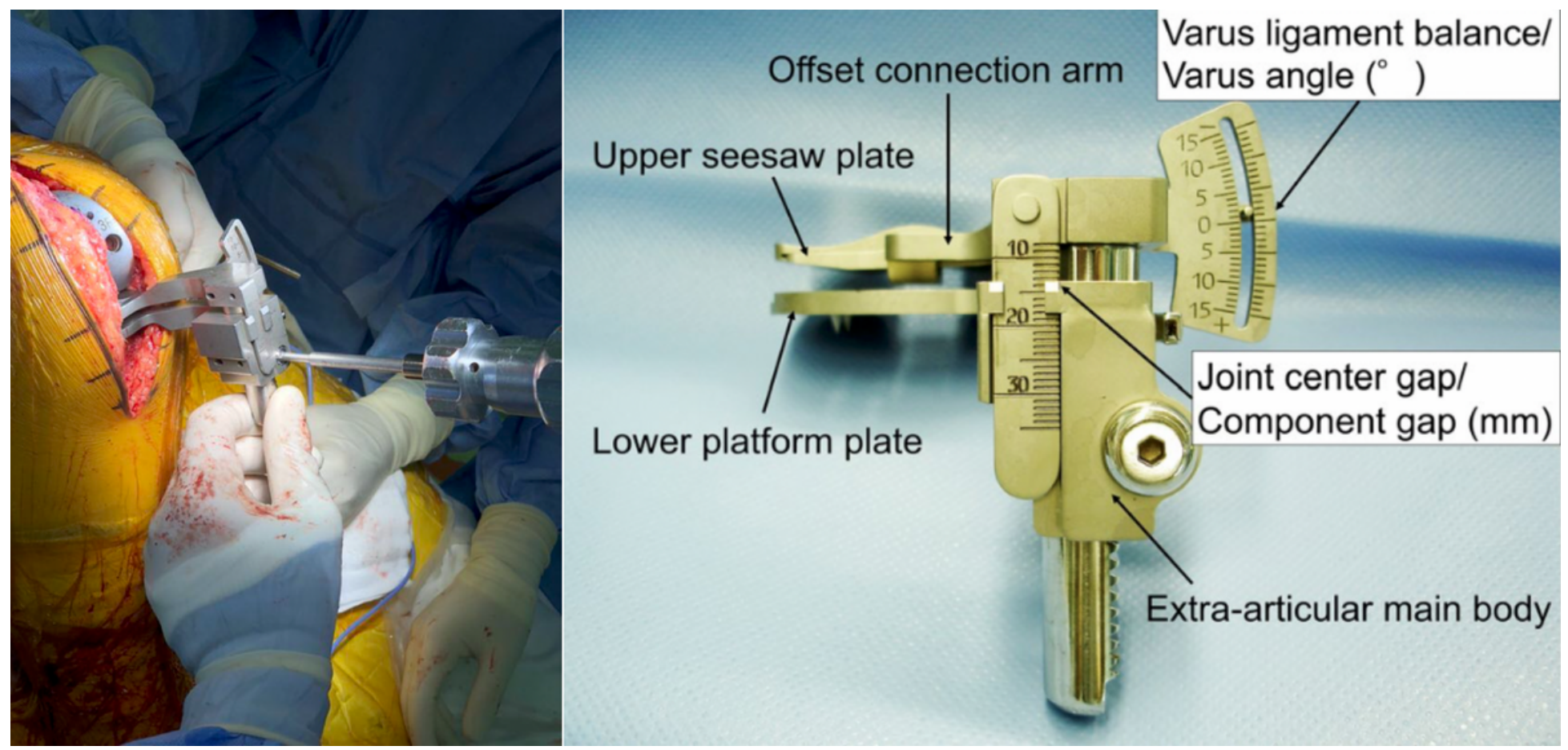

\section{Figure 3}

The Offset-Repo-Tensor and the Torque Wrench 


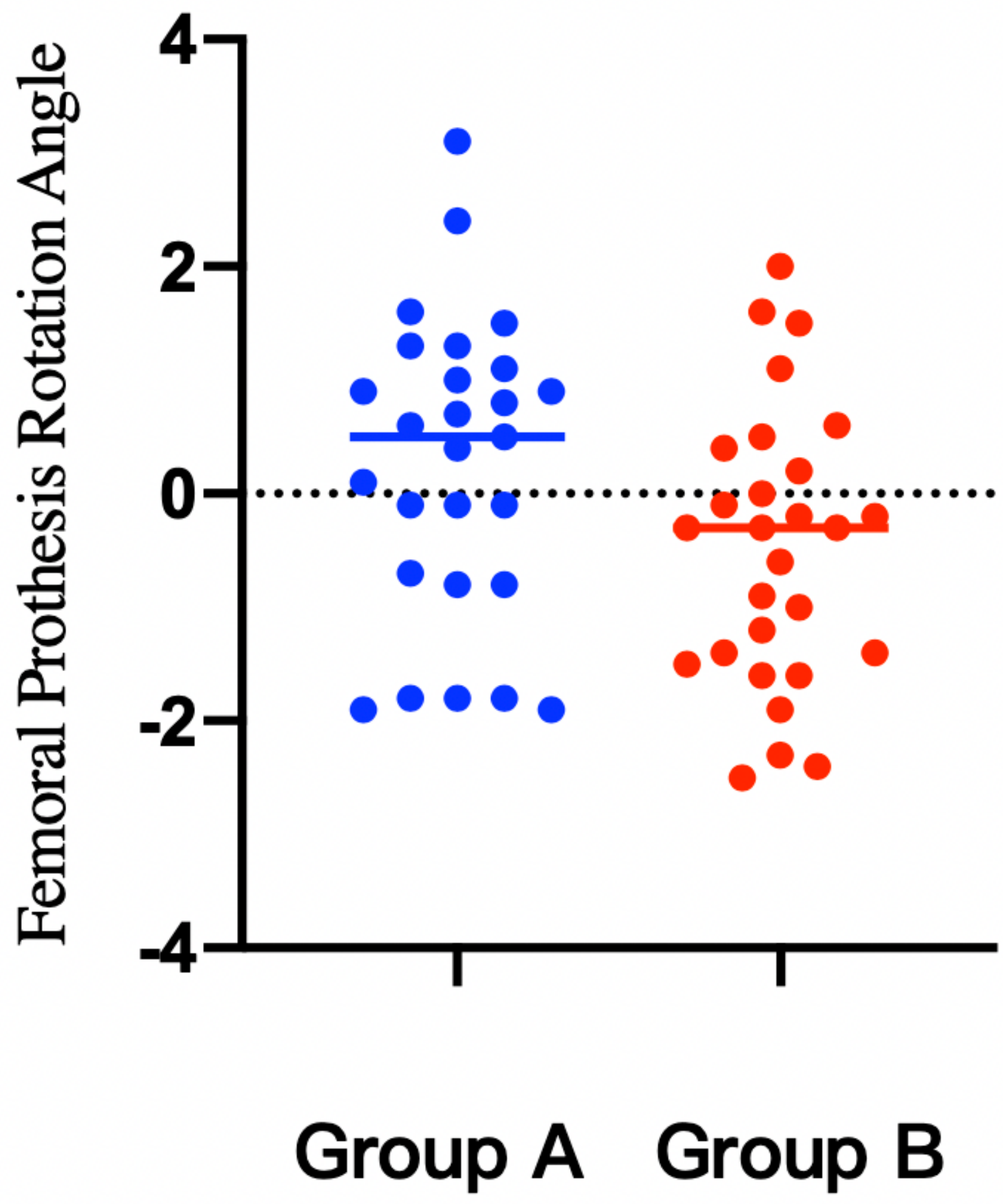

Figure 4

Scatter plot of femoral prothesis rotation angle 


\section{KSS评分}

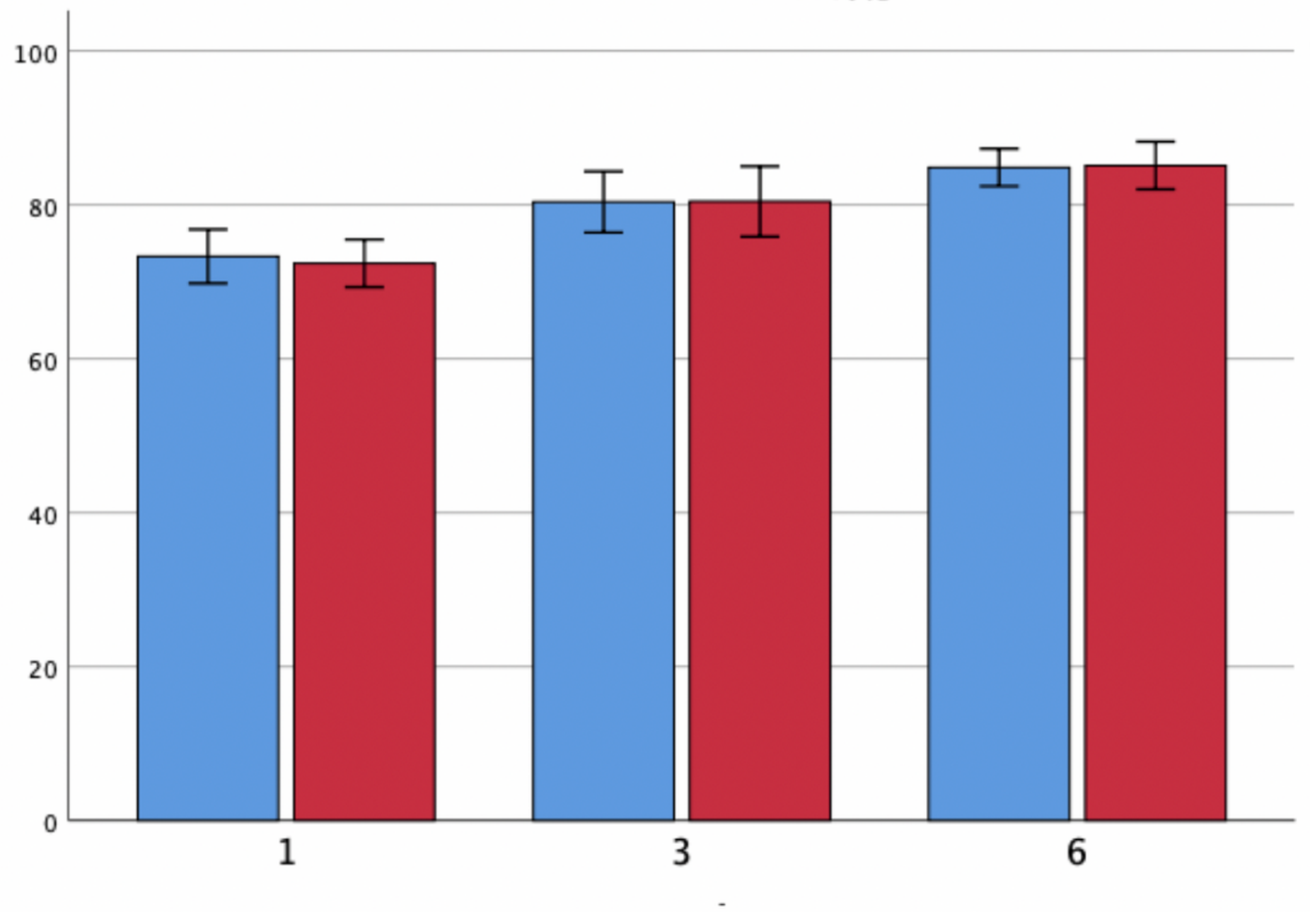

Figure 5

Comparison of postoperative Knee Society Scores between the two groups. 


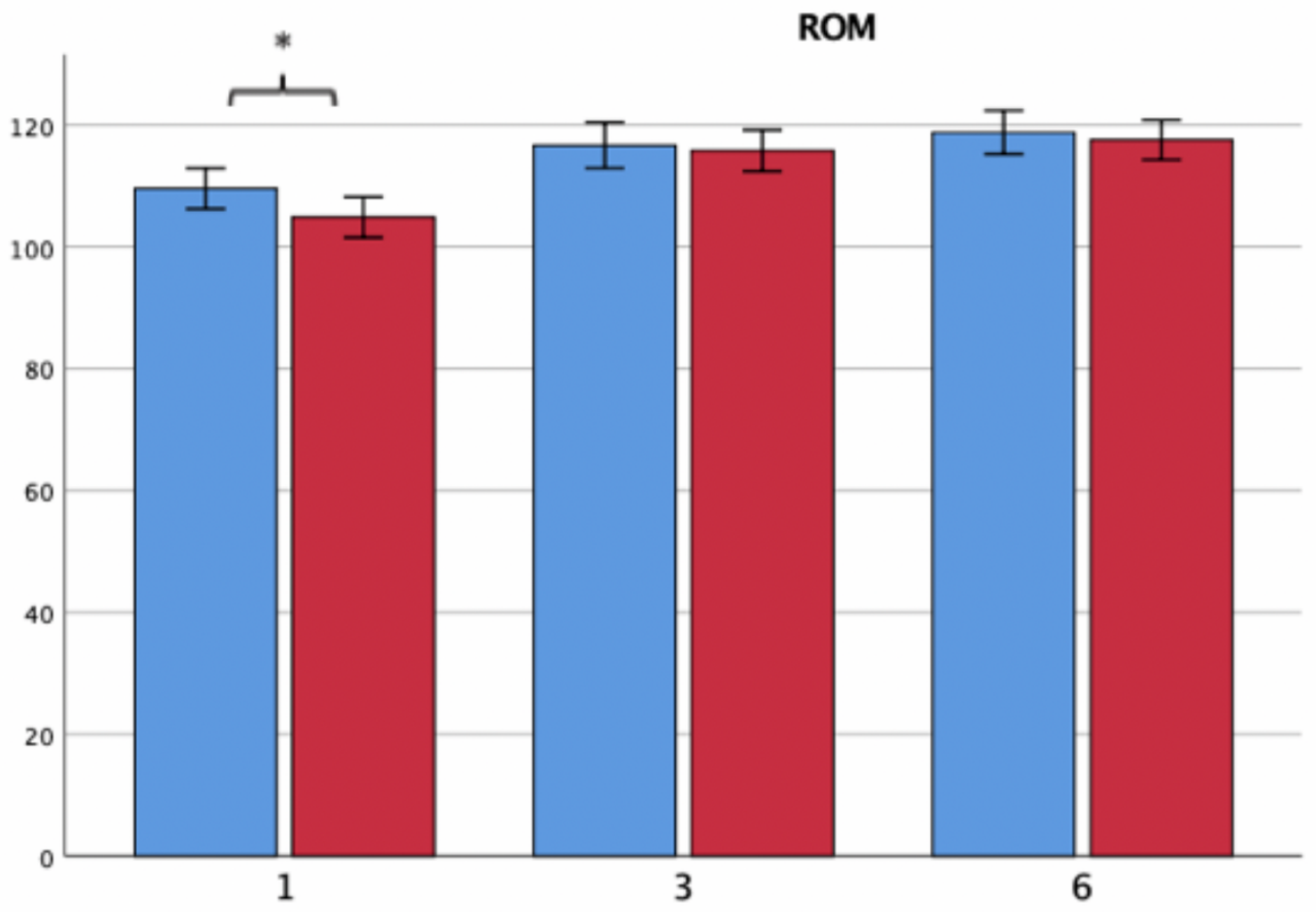

Figure 6

Comparison of postoperative range of flexion between the two groups. 


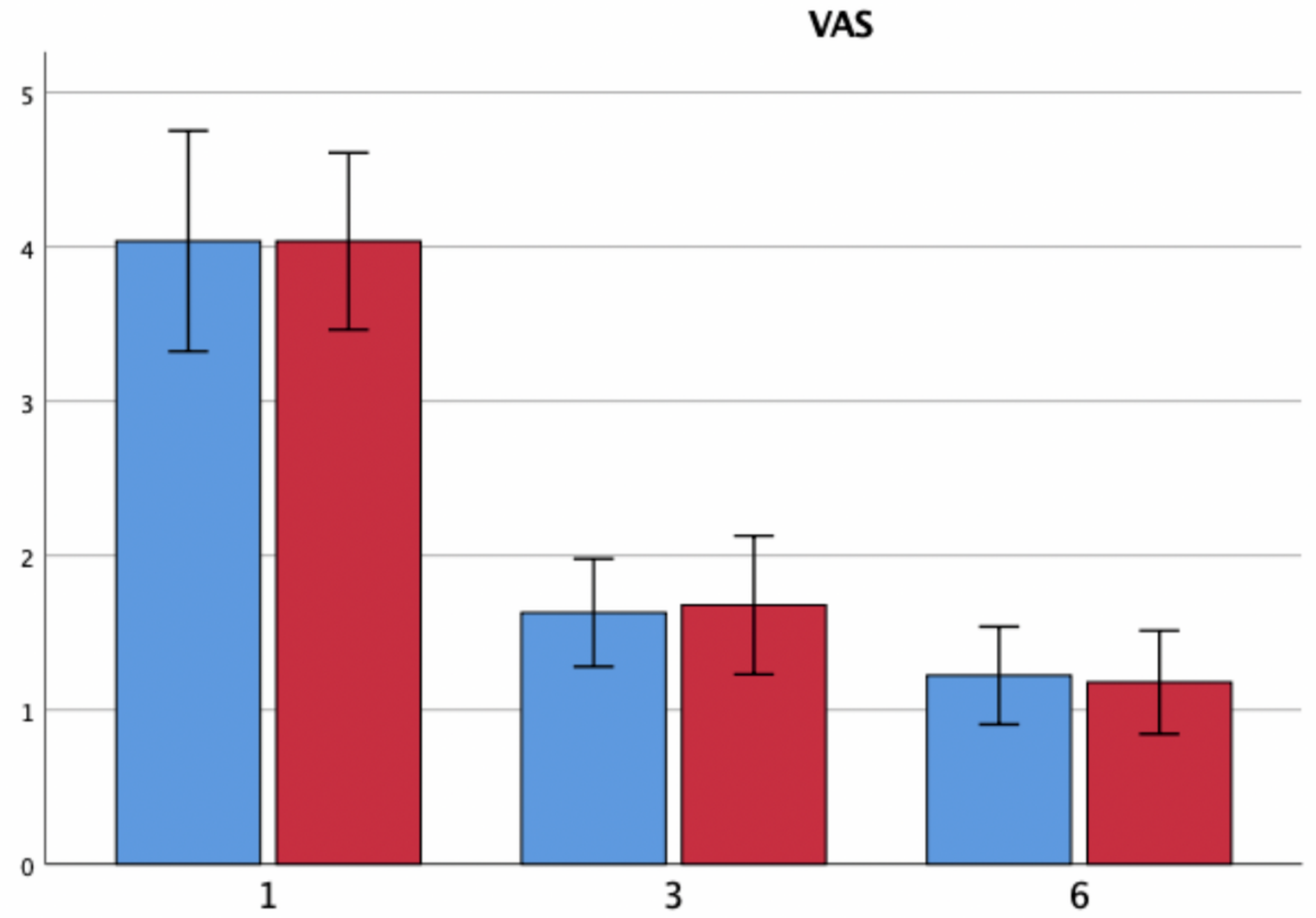

Figure 7

Comparison of postoperative pain scores (visual analog scores) between the two groups 


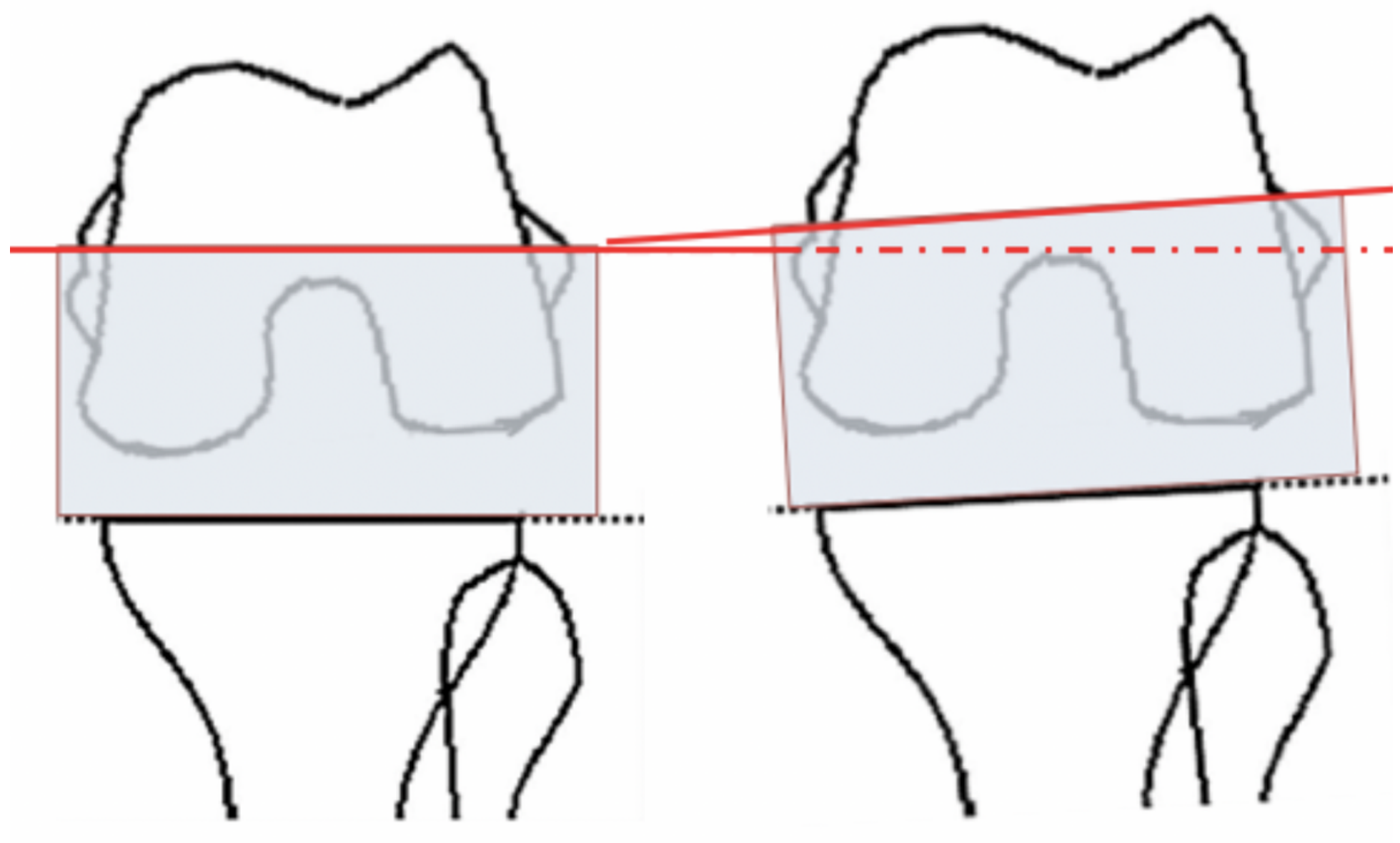

Reduction

Subluxation

Figure 8

The tibial plateau was taken as the reference to perform osteotomy of the femoral posterior condylar with the gap-balance technique. So the scribe line recorded by using the matching rectangle parallel to tibial plateau was more internal and this eventually caused the internal installment of the femoral prothesis 